\title{
Características de las residencias de terapia intensiva pediátrica de la República Argentina. Encuesta nacional
}

\author{
Characteristics of pediatric intensive care residency programs \\ in Argentina. A national survey
}

\author{
Dr. Martín Truszkowski ${ }^{a}$, Dr. Rodolfo P. Moreno ${ }^{a}$, Dra. Silvia N. Santos ${ }^{a}$, \\ Dr. Guillermo E. Moreno ${ }^{a}$, Dr. Thomas Iolstera, Dr. Alejandro Siaba Serrate ${ }^{b}$, \\ Dr. Luis Landry a,b, Dra. María Elena Ratto ${ }^{b}$,Dr. Daniel Rufach ${ }^{a}$, \\ Dra. Analía Fernández ${ }^{b}$ Dr. Juan C. Vassallo ${ }^{a}$ Dr. Daniel Buamscha ${ }^{a}$, \\ Dr. Gustavo Debaisi ${ }^{a}$ Dr. Horacio Yulitta ${ }^{c}$ y Grupo de Investigación Clínica y \\ Epidemiológica en Terapia Intensiva Pediátricad
}

\section{RESUMEN}

Introducción. La residencia de terapia intensiva pediátrica (TIP) tiene pocos años de desarrollo en nuestro país. Conocer su situación brinda la posibilidad de establecer estrategias para contribuir al desarrollo y capacitación de profesionales.

Objetivos. 1) Describir las características de las residencias de TIP del país. 2) Evaluar si existen características que se relacionen con una mayor ocupación de las vacantes.3) Explorar la inserción laboral en el hospital formador de los residentes. Diseño. Descriptivo, observacional. Encuesta nacional.

. Comité Nacional de Emergencias y Cuidados Críticos, Sociedad Argentina de Pediatría.

b. Capítulo Terapia Intensiva Pediátrica, Sociedad Argentina de Terapia Intensiva.

c. Consejo de Acreditación de Espacios de Formación (CAEF), Sociedad Argentina de Pediatría.

d. Miembros del Grupo de Investigación Clínica y Epidemiológica en Terapia Intensiva Pediátrica (véase en el Anexo 1, en formato electrónico).

Correspondencia: Dr. Rodolfo Pablo Moreno: morenopablorod@ hotmail.com

Financiamiento: Ninguno.

Conflicto de intereses: Ninguno que declarar.

Recibido: 23-1-2015

Aceptado: 11-5-2015

\section{INTRODUCCIÓN}

Las unidades de cuidados intensivos pediátricos (UCIP) han tenido un importante desarrollo en los últimos treinta años, resultado de la combinación de los siguientes factores: conocimiento de la fisiopatología del paciente crítico, avances tecnológicos y crecimiento en la ingeniería biomédica, avance de la ética profesional y vocación de los profesionales por esta especialidad. ${ }^{1,2}$

El sistema de residencias es considerado el mejor modelo para alcanzar la especialización en el equipo de salud. ${ }^{3}$ En este modelo de capacitación intensiva, los servicios de salud se transforman en instituciones formadoras, que cubren el doble rol de espacios de trabajo y formación. ${ }^{4}$

La residencia de terapia intensiva pediátrica (TIP) tiene pocos años de desarrollo en nuestro país. Conocer cuál es la situación de las residencias de TIP brinda la posibilidad de establecer estrategias que permitan contribuir al desarrollo y capacitación de profesionales que le puedan brindar a la población pediátrica los cuidados que demanda nuestra sociedad. En nuestro país, no hay estudios publicados acerca de las residencias de TIP.

\section{OBJETIVOS}

- Describir las características de las residencias de TIP en el país.

- Evaluar si existen características 
de las residencias que se relacionen con una mayor cobertura de las vacantes asignadas.

- Explorar la inserción laboral en el hospital formador de los residentes de TIP.

\section{POBLACIÓN Y MÉTODOS \\ Metodología de trabajo}

Se invitó a participar a todas las residencias de TIP del país a través de una nota dirigida al jefe del Servicio, en la que se detallaban las condiciones de participación, objetivos, fecha de inicio y fin del relevamiento y definiciones operativas de las variables.

Los autores fueron responsables de realizar el relevamiento en las residencias, recopilar los datos obtenidos y contactarse con las diferentes TIP.

La participación de los jefes del Servicio tuvo los siguientes alcances: recolección y envío de los datos a representantes de la Sociedad Argentina de Pediatría (SAP) y la Sociedad Argentina de Terapia Intensiva (SATI).

\section{Diseño}

Descriptivo, observacional, transversal. Encuesta nacional.

\section{Criterios de inclusión}

Las residencias de TIP de la República Argentina que se encontraban funcionales al momento de la encuesta realizada entre el $1 / 4 / 2014$ y el 31/5/2014.

\section{Definiciones}

- UCIP de gran volumen: tienen $\geq 8$ camas y $\geq 400$ ingresos anuales. ${ }^{1,5-7}$

- Acreditación de residencias: es un proceso de armonización de los criterios mínimos para la formación, que establece una base común que busca asegurar una formación de calidad en todo el país y genera lineamientos curriculares comunes. El Sistema Nacional de Acreditación de Residencias del Equipo de Salud está regulado por las Resoluciones Ministeriales 450/06 y 1342/07. ${ }^{8,9}$ Cuenta con la adhesión de todas las jurisdicciones por su aprobación por el Consejo Federal de Salud.

- Inserción laboral: contrato de empleo y/o guardia fija.

\section{Diseño de la encuesta}

Fue realizado por el Comité Nacional de Emergencias y Cuidados Críticos de la SAP y el Capítulo Terapia Intensiva Pediátrica de la SATI, utilizando como fuente el Marco de Referencia para las Residencias, Especialidad Terapia Intensiva Infantil ${ }^{10}$ el Instrumento de Evaluación de Residencias ${ }^{8}$ y los Estándares Nacionales para Acreditación de Residencias. ${ }^{9}$

\section{Análisis estadístico}

Las variables continuas se presentaron como mediana e intervalo intercuartílico (interquartile range; IQR, por sus siglas en inglés). Las variables categóricas se expresaron como porcentajes e intervalo de confianza del 95\% (IC 95\%) y se compararon con el Test de Chi².

Se realizó un análisis de regresión logística univariado para evaluar la relación entre las variables independientes y el evento de interés y, posteriormente, un análisis de regresión logística multivariado.

Se seleccionaron para ingresar al modelo multivariado las variables que presentaron $p \leq$ 0,2 asociada al test de Wald, y quedaron aquellas con AES (test de Wald: $\mathrm{p}<0,05$ ).

Se evaluó la presencia de confundidores en un proceso iterativo hasta excluir todas las variables biológica y estadísticamente irrelevantes. Las medidas de efecto se informaron como odds ratio (OR) con sus respectivos intervalos de confianza (IC) del 95\%. Se evaluó la robustez del modelo con el test de Hosmer-Lemeshow.

También se realizó la búsqueda de probables valores atípicos y potenciales influyentes.

\section{Aspectos éticos}

El acceso a la información recabada contó con los recaudos establecidos por las normas éticas y legales (Ley 25326). Los datos obtenidos no se utilizaron para otros fines y se preservó la identidad de las UCIP mediante disociación (datos codificados o reversiblemente disociados).

Este estudio fue aprobado por los comités institucionales de los hospitales participantes.

No existió ningún tipo de financiamiento ni conflictos de intereses para declarar.

\section{RESULTADOS \\ Perfil de los hospitales participantes}

Se invitó a las 32 residencias de TIP a participar; 31 respondieron afirmativamente y enviaron sus informes. El $87 \%(n=27)$ eran públicas y el $13 \%(n=4)$, privadas. Estaban situadas del siguiente modo: 14 hospitales pediátricos, 12 hospitales generales, 5 hospitales materno-infantiles. 


\section{Características generales de las unidades de cuidados intensivos pediátricos}

En 19 instituciones, existían, además, unidades de cuidados intermedios.

La mediana de camas de las UCIP fue 10 (IQR 8-15) (privadas: 9 camas, IQR 4-13; públicas: 11 camas, IQR 7-15). La mediana de ingresos anuales fue 378,70 (IQR 200-470).

Las UCIP de gran volumen fueron $11 / 31$ (36,67\%; IC 85\%: 0,18-0,55).

\section{Características funcionales de las unidades de cuidados intensivos pediátricos}

Todas internaron patología médica, quirúrgica, traumatológica.

En 21 UCIP, se atendieron pacientes con trauma de cráneo grave. La mediana de neurocirugías anual fue 19 (IQR 7-42).

En 20 UCIP, se internaron posoperatorios de cirugías cardiovasculares y 15 recuperaron cirugías cardiovasculares neonatales. La mediana de cirugías cardiovasculares anual fue 25 (IQR 0-95).

La Tabla 1 muestra las terapéuticas que realizaban las UCIP.

TABLA 1. Terapéuticas utilizadas en las unidades de cuidados intensivos pediátricos

\begin{tabular}{|c|c|}
\hline Modalidad & Número de UCIP \\
\hline $\begin{array}{l}\text { Asistencia ventilatoria } \\
\text { mecánica convencional }\end{array}$ & 31 \\
\hline Ventilación de alta frecuencia & 24 \\
\hline Oximetría de pulso & 31 \\
\hline Oximetría de pulso(\#) & 29 \\
\hline Monitoreo respiratorio & 30 \\
\hline Monitoreo cardíaco & 31 \\
\hline Monitoreo cardíaco ${ }^{(\#)}$ & 30 \\
\hline Monitoreo de $\mathrm{PA}^{(\mathrm{a})}$ invasiva & 31 \\
\hline Monitoreo de $\mathrm{PA}^{(\mathrm{a})}$ invasiva $^{(\#)}$ & 27 \\
\hline Monitoreo de PVC ${ }^{(b)(\#)}$ & 31 \\
\hline Marcapasos externo transitorio & 28 \\
\hline $\mathrm{ECMO}^{(\mathrm{c})}$ & 4 \\
\hline Diálisis peritoneal & 30 \\
\hline Hemofiltración & 14 \\
\hline Monitoreo de PIC (d) & 25 \\
\hline
\end{tabular}

UCIP: unidades de cuidados intensivos pediátricos.

(\#) Simultáneamente en todas las camas de la UCIP.

(a) PA: presión arterial.

(b) PVC: presión venosa central.

(c) ECMO: oxigenación con membrana extracorpórea.

(d) PIC: presión intracraneana.

\section{Registros médicos}

Los ingresos anuales fueron registrados por 29/31 TIP; 27 UCIP poseían registro informatizado de datos.

Las variaciones de la mortalidad en relación con los puntajes pronósticos de gravedad (puntaje de PIM2) se realizaron en 22/31.

\section{Características de las residencias}

La mediana de antigüedad fue 6 años (IQR 4-15) y la mediana de duración, 2 años (IQR 2-3).

Dependencia: $15(48,39 \%)$ provinciales, 7 $(22,58 \%)$ nacionales, $4(12,90 \%)$ municipales, $1(3,22 \%)$ universitaria, $4(12,90 \%)$ privadas. Son sede de carrera universitaria de médico especialista en TIP 12/31.

Las residencias pertenecían: 8 a la provincia de Buenos Aires, 6 a la Ciudad Autónoma de Buenos Aires (CABA), 4 a Santa Fe, 2 a Córdoba y el resto, una a cada una de las provincias de Catamarca, Chaco, Corrientes, Entre Ríos, La Rioja, Mendoza, Misiones, Neuquén, Salta, San Juan y Tucumán.

Una residencia (sector público) no tenía programa de residencia y 2 no tenían reglamento. No hay normas y/o criterios de atención en 9/31.

Fueron acreditadas por el Ministerio de Salud de la Nación 5 residencias.

\section{Ingreso}

Se utilizó examen escrito en 30; una TIP privada reportó otra modalidad.

\section{Vacantes}

En el año 2013, hubo 65 vacantes disponibles; 61 de ellas, en el sector público. La mediana de vacantes por residencia fue 2 (IQR 1-3).

Las vacantes aumentaron en los últimos 4 años y la ocupación disminuyó de 59\% en 2009 a 30\% en 2013; hay residencias que nunca ocuparon las vacantes disponibles (Figura 1). En el período 2009-2013, se formaron 94 residentes; el 60\% tuvo inserción laboral en la TIP que lo formó.

\section{Actividad del residente}

Distribución de la carga horaria semanal: $1^{\text {er }}$ año, mediana de 60 horas (IQR 48-76); $2^{\circ}$ año, 58 horas (IQR 50-68); y $3^{\text {er }}$ año, 51 horas (IQR 40-62).

La distribución de la actividad de los residentes se muestra en la Figura 2.

Guardias (mediana) por mes: $1^{\mathrm{er}}$ año, 6 (IQR 6-7) (en 5 UCIP del sector público, el número fue 8); $2^{\circ}$ año, 5 (IQR 5-6); $3^{\text {er }}$ año, 4 (IQR 4-6). En todas las UCIP, las guardias son supervisadas por médicos especialistas en TIP. 
El descanso posguardia no fue otorgado en 11/31 TIP, todas del sector público.

\section{Rotaciones externas}

Se realizaron en 25 residencias. Los sitios de rotación elegidos por los residentes fueron UCIP cardiovasculares (19/25), otras UCIP polivalentes $(14 / 25)$, TIP de quemados $(4 / 25)$.

\section{Docencia}

Contaban con jefe de residentes $8 / 31$ y con instructor, 15/31. Hubo 13/31 TIP (11 públicas, 2 privadas) que no contaban con ninguno de los dos.

En 26 TIP (83,87\%), existía un responsable de la residencia y $25(80,65 \%)$ tenían médicos asignados a la docencia. La mediana de docentes por servicio fue 3 (IQR 1-10). En 8/31 UCIP, los responsables del programa y/o los docentes recibieron formación pedagógica.

El programa estuvo adecuado al marco de referencia nacional en 17/31.

Todas ofrecieron clases, con una frecuencia promedio de 2 por semana. Todas realizaban pase diario y pase de guardia, ambos supervisados por médicos de planta y/o guardia.

Se realizaban ateneos bibliográficos en $28(90,32 \%)$, anatomoclínicos en $25(80,64 \%)$, de mortalidad en 15 (48,38\%). En $27 / 31$, se supervisaban las historias clínicas.

Hubo clases de lectura crítica en 16/31 y curso de reanimación cardiopulmonar en todas.
FIGURA 1. Relación entre las vacantes disponibles y la ocupación de cargos de residencias

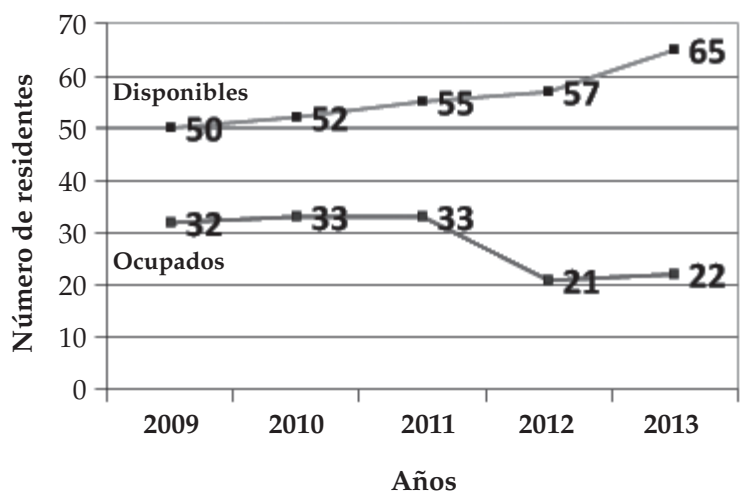

\section{Evaluaciones}

Los residentes fueron evaluados 3 veces por año (IQR 2-4). Tipos de evaluaciones: 29 (93,54\%) observaciones directas por médicos de planta y/o jefes, 26 (83,87\%) exámenes escritos, 16 (51,61\%) exámenes orales, $15(48,38 \%)$ listas de cotejo. Solo 2/31 utilizaban ECEO y ninguna SCRIPT o MINICEX.

En $25 / 31$, había examen al finalizar la residencia y 20/31 registraban los procedimientos.

\section{Investigación}

Se realizaron trabajos de investigación en 24/31. En $1 / 31$, hubo clases de investigación y $19 / 31$ contaban con tutores.

Solo 8/31 publicaron los trabajos producidos. La mayoría fueron presentados en jornadas hospitalarias $(n=16)$ y congresos nacionales $(n=20)$.

\section{Marco laboral de las residencias}

En 11/31, los residentes tenían cobertura del seguro médico de mala praxis y, en 26/31,

Figura 2. Distribución de tiempo del programa de residencia según las actividades

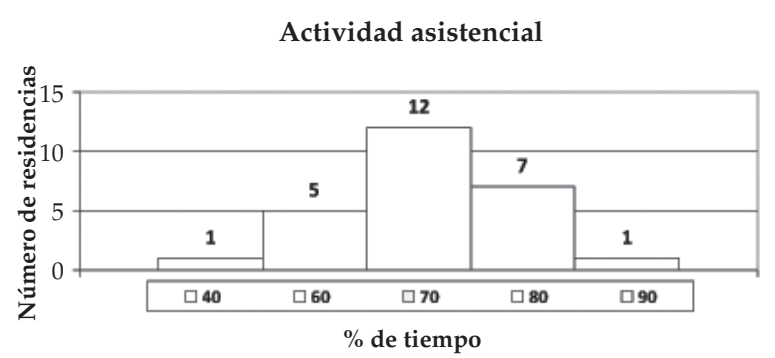

Actividad docente

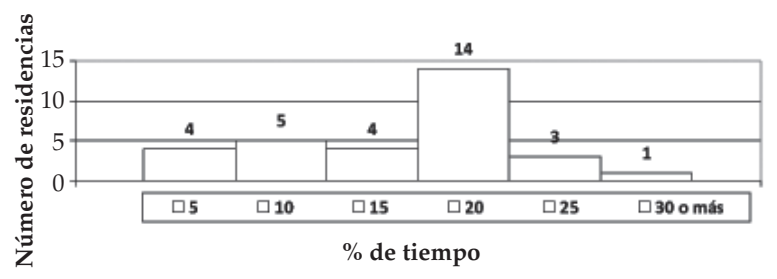

Investigación

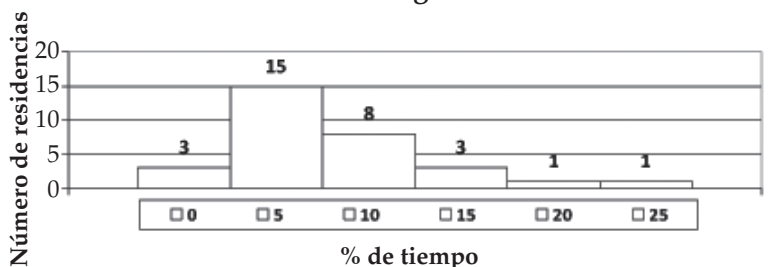


aseguradora de riesgo de trabajo (ART). Brindaban cobertura del seguro de salud 28 residencias $(90,32 \%)$. El 100\% ofrecían vacaciones, licencia por maternidad, licencia por enfermedad y para asistencia a congresos.

Proveían uniforme laboral 8/31 UCIP.

\section{Factores predictores de cobertura de vacantes}

Se realizó un análisis bivariado en búsqueda de variables relacionadas con la variable "cobertura de vacantes $>60 \%$ ", definida como residencias que ocupaban $>60 \%$ de las vacantes asignadas en el período 2009-2013. Se encontró significancia estadística entre la variable de interés con TIP de mayor volumen $(p=0,001)$, realización de trabajos de investigación $(p=0,012)$, cobertura del seguro de mala praxis por el financiador $(\mathrm{p}=0,010) \mathrm{y}$ cobertura social $(\mathrm{p}=0,032)$ (Tabla 2$)$.

Se realizó un análisis de regresión logística multivariado para identificar variables que pudieran predecir en forma independiente la variable de interés "cobertura de vacantes $>60 \%$ ". Ingresaron al modelo variables con $p<0,2$ (ingresos anuales $>400$, cobertura de seguro de mala praxis, realización de trabajos de investigación, cobertura social, cobertura de ART, presencia de instructor, presencia de médicos de guardia con horas de planta). Se identificó la variable "ingresos anuales $>400$ " como predictora en forma independiente de la cobertura de vacantes $>60 \%$; las restantes presentaron $p$ no significativa e intervalos de confianza muy amplios.

TABla 2. Análisis bivariado de ocupación de vacantes $>60 \%$

\begin{tabular}{lc}
\hline VARIABLES & Valor de P \\
\hline UCIP > 400 ingresos/año & 0,00 \\
Realización de trabajos de investigación & 0,012 \\
Seguro de mala praxis & 0,010 \\
Cobertura social & 0,032 \\
Residencia articulada & 0,060 \\
Dependencia ${ }^{(\#)}$ & 0,084 \\
Presencia de médicos de planta & 0,069 \\
Jefe de servicio certificado & 0,085 \\
Presencia de jefe o instructor de residentes & 0,095 \\
Descanso posguardia & 0,297 \\
\hline
\end{tabular}

(\#) Nacional, provincial, municipal.

\section{DISCUSIÓN}

La residencia, como formación de posgrado, integra los conocimientos con la práctica en el medio hospitalario e intenta dar respuesta a las necesidades de salud. ${ }^{4,11}$ El residente tiene una doble oportunidad de aprendizaje, tanto de un área de conocimientos como del desarrollo del trabajo en situaciones reales con diferentes escenarios. $^{4,12}$

En el país, funcionan 32 residencias de TIP, la mayoría con escaso número de residentes en relación con las vacantes y con el número de camas. Esta realidad se contrapone con la de Estados Unidos, donde existen 60 residencias con un promedio de 7 residentes por centro. ${ }^{12}$ Estos datos muestran que, en Argentina, el número de médicos en formación puede no ser el adecuado.

El Ministerio de Salud de la Nación sugiere que el número de camas de TIP no debe ser menor de seis $;^{13}$ sin embargo, observamos TIP sedes de residencias con menos de 6 camas y gran variación en los recursos humanos y tecnológicos. Las diferencias en la adecuación de la estructura organizativa y la distribución de las residencias en relación con los egresos del sector y con las necesidades del sistema deberían estudiarse. ${ }^{11}$ El desafío para el futuro será establecer una formación equivalente en todo el país con estándares mínimos del perfil profesional ajustados al marco de referencia nacional. ${ }^{10}$

La mayoría de las residencias poseen programa, ${ }^{10}$ pero la existencia de residencias sin programas ni reglamentos es una observación para destacar. Nuestras sociedades deberían poner en marcha esfuerzos conjuntos de asesoramiento para asegurar el cumplimiento de los objetivos docentes de todo el programa y no solo la participación de los residentes en ámbitos de gran demanda asistencial. ${ }^{14,15}$

En Estados Unidos, los residentes de TIP dedican un $50 \%$ de las horas semanales de trabajo a la investigación. ${ }^{12}$ En las residencias de TIP evaluadas, menos del $60 \%$ incluyen clases o talleres de investigación, actividad a la que se le otorga una mínima carga horaria. Este hecho no siempre es percibido por los residentes como una carencia, ${ }^{16}$ tal como sucede con otros aspectos de la medicina actual, como la comunicación en situaciones complejas o la gestión de recursos.

Una residencia necesita que la tarea asistencial sea supervisada por personal capacitado, y los docentes involucrados tienen la obligación de asegurar que todos los residentes adquieran 
las habilidades necesarias para ejercer su especialidad adecuadamente. ${ }^{12,17}$

La evaluación del desempeño tiene por fin comprobar de modo sistemático el logro de los resultados previstos en los objetivos especificados. ${ }^{15,18}$ Sin embargo, al analizar las residencias, vimos que existía una gran variación, por lo que es otro punto para trabajar para establecer estándares equivalentes.

Respecto a la carga laboral, los residentes de TIP argentinos trabajan más horas que en otros países de América Latina, ${ }^{3}$ pero menos que en Estados Unidos. ${ }^{19}$ El Accreditation Council for Graduate Medical Education International recomendó disminuir horas de trabajo médico de los residentes, aumentar la supervisión y el enfoque basado en la seguridad del paciente..$^{20,21}$ En Francia, el trabajo de los residentes se restringió a 52,5 horas semanales. ${ }^{22}$ El Ministerio de Salud de la Nación establece que los residentes deberán cumplir no menos de 48 horas semanales y realizar no más de 8 guardias mensuales. ${ }^{4}$

Existe evidencia de que la carga laboral excesiva y la falta de distribución equitativa de las funciones llevan al burnout o síndrome de desgaste profesional ${ }^{19,23,24}$ y producen elevados índices de despersonalización y agotamiento emocional en los residentes. ${ }^{25,26} \mathrm{~K}$. E. Fletcher et al. ${ }^{23}$ sugirieron que la calidad de vida de los residentes puede mejorarse limitando las horas de trabajo; el impacto de esta reducción sobre la educación permanece desconocido. En Argentina, la prevalencia de burnout en los residentes es del 20\%. ${ }^{24}$ Para su prevención, es conveniente mejorar las condiciones objetivas de trabajo, ${ }^{27}$ contar con supervisión efectiva y no continuar las actividades después de las guardias. ${ }^{2}$ Sin embargo, los intensivistas pediátricos no perciben beneficios para la seguridad del paciente, que se lograrían al disminuir las horas de trabajo de los residentes. ${ }^{28,29}$

El número de vacantes ocupadas de las residencias de TIP ha disminuido en los últimos años. En nuestro medio, existen otros programas de formación: las becas de perfeccionamiento en TIP con acreditación académica universitaria. Estos sistemas de posgrado se ofrecen, incluso, en algunas TIP, cuyas residencias fueron parte de este estudio. Es probable que estas becas tengan un posible impacto en la reducción de la cobertura de vacantes; sin embargo, de haber suficientes interesados en realizar la especialidad, se cubrirían los puestos de becas y de residencias. La falta de intensivistas pediátricos es una realidad global, ${ }^{30-32}$ cuya causa es multifactorial.
El objetivo primario del sistema de residencias debe ser asegurar la formación de médicos que puedan proporcionar a sus pacientes un cuidado clínico excelente y que desarrollen su especialidad durante largo tiempo. ${ }^{33}$ Las conclusiones evidencian la necesidad de reorientar las políticas de recursos humanos en salud y la formación de especialistas en particular.

A pesar del carácter esencialmente descriptivo del trabajo, estamos convencidos de la importancia de este tipo de estudios a fin de suministrar información que permita mejorar la capacitación en TIP, facilitar la toma de decisiones en la asignación de recursos basadas en datos locales y reafirmar la importancia de estimular la elección de la subespecialidad por los médicos jóvenes.

\section{CONCLUSIONES}

1. Se registra un importante déficit en la ocupación de cargos de las residencias en TIP.

2. El número de residencias acreditadas por el Ministerio de Salud de la Nación es escaso.

3. Las UCIP con mayor número de ingresos se asociaron a una mejor cobertura de las vacantes de residencia.

4. Más de la mitad de los residentes se insertaron laboralmente en la TIP que los formó.

\section{REFERENCIAS}

1. Namachivayam P, Shann F, Shekerdemian L, Taylor A, et al. Three decades of pediatric intensive care: Who was admitted, what happened in intensive care, and what happened afterward. Pediatr Crit Care Med 2010;11(5):549-55.

2. RoqueJ.Cuidados intensivos pediátricos: pasado, presente y futuro. Rev Chil Pediatr 2013;84(3):249-53.

3. Organización Panamericana de la Salud. Área de Sistemas de Salud basados en la Atención Primaria de Salud. Residencias médicas en América Latina. Washington DC; 2011.

4. Argentina. Ministerio de Salud. Dirección Nacional de Capital Humano y Salud Ocupacional. Sistema nacional de acreditación de residencias del equipo de salud. Normativa, guías y estándares a 7 años de su creación. Ciudad Autónoma de Buenos Aires; 2014.

5. Nipshagen MD, Polderman KH, DeVictor D, Gemke NJ. Pediatric intensive care: result of a European survey. Intens Care Med 2002;28(12):1797-803.

6. Odetola FO, ClarkSJ, Freed GL, Bratton SL, et al. A national survey of pediatric critical care resources in the United States. Pediatrics 2005;115(4):e382-6.

7. Köroğlu TF, Atasever S, Duman M. A survey of pediatric intensive care services in Turkey. Turk J Pediatr 2008;50(1):12-7.

8. Argentina. Ministerio de Salud. Dirección Nacional de Capital Humano y Salud Ocupacional. Instrumento de Evaluación de Residencias/2010. Buenos Aires, 2010. [Acceso: 2 de marzo de 2014]. Disponible en: http: / www. msal.gov.ar/residencias/images/stories/descargas / acreditaciones/adjuntos/instrumento $\% 20$ residencias $\% 20$ 2010.pdf. 
9. Argentina. Ministerio de Salud. Dirección Nacional de Capital Humano y Salud Ocupacional. Estándares Nacionales para Acreditación de Residencias. Buenos Aires, 2010. [Acceso: 2 de marzo de 2014]. Disponible en: http://www.msal.gov.ar/residencias/images/stories/ descargas/acreditaciones/adjuntos/estandares $\% 20$ nacionales \%20para\%20acreditacion \%20de \%20residencias. pdf.

10. Argentina. Ministerio de Salud. Sistema Nacional de Residencias del Equipo de Salud. Marco de Referencia para la Formación en Residencias Médicas. Especialidad Terapia Intensiva Infantil. Buenos Aires, 2013. [Acceso: 2 de mayo de 2014]. Disponible en: http:/ /www.msal.gov.ar/ residencias/images/stories/descargas/acreditaciones/ adjuntos/terapia_intensiva_infantil_mr.pdf.

11. Borrell Bentz RM. La educación médica de posgrado en Argentina: el desafío de una nueva práctica educativa. Buenos Aires: Organización Panamericana de la Salud; 2005.

12. Morrison WE, Helfaer MA, Nadkarni VM. National survey of pediatric critical care medicine fellowship clinical and research time allocation. Pediatr Crit Care Med 2009;10(3):397-9.

13. Resolución 747/2014. Directrices de Organización y Funcionamiento de las Unidades de Cuidados Intensivose Intermedios Pediátricos en Establecimientos Asistenciales. Boletín Oficial de la República Argentina, Buenos Aires, Argentina, 22 de mayo de 2014.

14. Bowen KA, Ball TM. Pediatricians' evaluations of their residency curriculum in emergency medicine. Pediatr Emerg Care 2003;19(2):79-83.

15. Busaniche J. La importancia de la evaluación de los residentes. ¿Qué, cómo y cuándo evaluar? Arch Argent Pediatr 2007;105(4):291-2.

16. Vázquez-MataG,Rodríguez-Elvira M,Rucabado-AguilarL, García-Alcántara A, et al. Los residentes en las unidades de cuidados intensivos, ¿cuál es su percepción de la formación que reciben? Educ Med 2011;14(3):189-94.

17. Grenvik A, Schaefer JJ $3^{\text {rd }}$. DeVita MA, Rogers P. New aspects on critical care medicine training. Curr Opin Crit Care 2004;10(4):233-7.

18. Galli A, CastroC. Programa de formación de formadores en ciencias de la salud. Volumen III. Buenos Aires: Asociación Amigos de la Facultad de Medicina; 1999.

19. Blum $A B$, Shea $S$, Czeisler $C A$, Landrigan $C P$, et al. Implementing the 2009 Institute of Medicine recommendations on resident physician work hours, supervision, and safety. Nat Sci Sleep 2011;3:47-85.
20. Nasca TJ, Day SH, Amis ES Jr. The new recommendations on duty hours from the ACGME Task Force. N Engl J Med 2010;363(2):e3.

21. Landrigan CP, Fahrenkopf AM, Lewin D, Sharek PJ, et al. Effects of the accreditation council for graduate medical education duty hour limits on sleep, work hours, and safety. Pediatrics 2008;122(2):250-8.

22. Woodrow SI, Segouin C, Armbruster J, Hamstra SJ, et al. Duty hours reforms in the United States, France, and Canada: is it time to refocus our attention on education? Acad Med 2006;81(12):1045-51.

23. Fletcher KE, Underwood W 3rd, Davis SQ, Mangrulkar RS, et al. Effects of work hour reduction on residents' lives: a systematic review. JAMA 2005;294(9):1088-100.

24. Catsicaris C, Eymann A, Cacchiarelli N, Usandivaras I. La persona del médico residente y el síndrome de desgaste profesional (burnout). Un modelo de prevención en la formación médica. Arch Argent Pediatr 2007;105(3):236-40.

25. López-Morales A, González-VelázquezF,Morales-Guzmán MI, Espinoza-Martínez CE. Síndrome de burnout en residentes con jornadas laborales prolongadas. Rev Med Inst Mex Seguro Soc 2007;45(3):233-42.

26. Mejía R, Diego A, Alemán M, Maliandi M, et al. Percepción de maltrato durante la capacitación de médicos residentes. Medicina (B Aires) 2005;65(4):295-301.

27. Gordon MB, Sectish TC, Elliott MN, Klein D, et al. Pediatric residents' perspectives on reducing work hours and lengthening residency: a national survey. Pediatrics 2012;130(1):99-107.

28. Freed GL, Dunham KM, Moran LM, Spera L. Resident work hour changes in children's hospitals: impact on staffing patterns and workforce needs. Pediatrics 2012;130(4):700-4.

29. Typpo KV, Tcharmtchi MH, Thomas EJ, Kelly PA, et al. Impact of resident duty hour limits on safety in the intensive care unit: a national survey of pediatric and neonatal intensivists. Pediatr Crit Care Med 2012;13(5):578-82.

30. Galván ME, Vassallo JC, Rodríguez SP, Otero P, et al. Síndrome de desgaste profesional (burnout) en médicos de unidades de cuidados intensivos pediátricos en la Argentina. Arch Argent Pediatr 2012;110(6):466-73.

31. Campos-Miño S, Sasbón JS, Von Dessauer B. Los cuidados intensivos pediátricos en Latinoamérica. Med Intensiva 2012;36(1):3-10.

32. Stromberg D. Pediatric cardiac intensivists: are enough being trained? Pediatr Crit Care Med 2004;5(4):391-2.

33. Peets A, Ayas NT. Restricting resident work hours: the good, the bad, and the ugly. Crit Care Med 2012;40(3):960-6. 


\section{ANEXO 1}

\section{MIEMBROS DEL GRUPO DE INVESTIGACIÓN CLÍNICA Y EPIDEMIOLÓGICA EN TERAPIA INTENSIVA PEDIÁTRICA}

Dra. María del Pilar Arias. Hospital General de Niños "Dr. Ricardo Gutiérrez",

Ciudad de Buenos Aires, Argentina.

Dra. Amparo Baldironi. Hospital Pediátrico del Niño Jesús, Córdoba, Argentina.

Dr. Alberto Blanco. Hospital Pediátrico "Juan Pablo II", Corrientes, Argentina.

Dra. Ana Carola Blanco. Hospital Nacional "Prof. Dr. Alejandro Posadas", Haedo, Argentina.

Dr. Daniel Gustavo Bergna. Hospital de Niños (Interzonal de Agudos Especializado en Pediatría)

"Superiora Sor María Ludovica", La Plata, Argentina.

Dra. María Alejandra Boretto. Sanatorio de Niños, Rosario, Argentina.

Dra. Carmen Colman. Hospital Pediátrico “Dr. Avelino Castelán”, Resistencia, Chaco, Argentina.

Dr. Pablo Eulmesekian. Hospital Italiano de Buenos Aires, Buenos Aires, Argentina.

Dra. Liliana Galleguillo. Hospital de la Madre y el Niño de La Rioja, La Rioja, Argentina.

Dr. Germán Kaltenbach. Hospital Provincial "Dr. Eduardo Castro Rendón”, Neuquén, Argentina.

Dra. Karina Fiquetron. Hospital de Alta Complejidad "El Cruce Néstor Carlos Kirchner",

Florencio Varela, Argentina.

Dr. Luis Folco. Hospital Público Materno Infantil, Salta, Argentina.

Dr. Macelo Itharte. Hospital Materno Infantil San Roque, Paraná, Argentina.

Dr. Walter Lorenz. Hospital Zonal General de Agudos "Lucio Meléndez", Adrogué, Argentina.

Dra. Liliana Luján. Hospital de Niños de la Santísima Trinidad, Córdoba, Argentina.

Dr. Lorenzo Marcos. Hospital del Niño Jesús, San Miguel de Tucumán, Tucumán, Argentina.

Dra. Marta Mosciaro. Hospital Pediátrico “Dr. Humberto J. Notti”, Mendoza, Argentina.

Dr. Rodolfo Pacce. Hospital de Niños de Santa Fe "Dr. O. Alassia”, Santa Fe, Argentina.

Dra. Alicia Panigazzi. Hospital Nacional "Prof. Dr. Alejandro Posadas", Haedo, Argentina.

Dr. Matías Penazzi. Hospital del Niño de San Justo, San Justo, Argentina.

Dr. Roberto Marcelo Pena. Hospital Municipal Materno Infantil de San Isidro

“Dr. C. Gianantonio", San Isidro, Argentina.

Dr. Javier Ponce. Hospital Público Descentralizado "Dr. Guillermo Rawson", San Juan, Argentina.

Dra. Graciela Rivello. Sanatorio Güemes, Buenos Aires, Argentina.

Dra. Graciela Romero. Hospital Interzonal de Niños "Eva Perón”, Catamarca, Argentina.

Dra. María Gabriela Rodríguez. Hospital de Clínicas UBA “José de San Martín”,

Ciudad de Buenos Aires, Argentina.

Dra. Silvia Susana Sáenz. Hospital de Niños de la Santísima Trinidad, Córdoba, Argentina.

Dra. Mariam Sarli. Hospital Nacional "Prof. Dr. Alejandro Posadas", Haedo, Argentina.

Dr. Gustavo Sciolla. Hospital Provincial de Rosario, Rosario, Argentina.

Dr. Ariel Segado. Hospital de Niños de Rosario "Víctor J. Vilela”, Rosario, Argentina.

Dr. Jorge Selandari. Sanatorio Güemes, Buenos Aires, Argentina.

Dr. Marcelo Terebiznik. Hospital Interzonal General de Agudos "Eva Perón",

San Martín, Argentina.

Dr. Silvio Fabio Torres. Hospital Universitario Austral, Pilar, Argentina.

Dra. Alicia Yunis. Hospital Público Provincial de Pediatría “Dr. Fernando Barreyro",

Posadas, Argentina. 